\title{
Politique
}

\section{Michel Crozier et l'étude des organisations}

\section{Stéphane Dion}

Numéro 12, automne 1987

Mouvements et acteurs

URI : https://id.erudit.org/iderudit/040570ar

DOI : https://doi.org/10.7202/040570ar

Aller au sommaire du numéro

Éditeur(s)

Société québécoise de science politique

ISSN

0711-608X (imprimé)

1918-6584 (numérique)

Découvrir la revue

Citer ce document

Dion, S. (1987). Michel Crozier et l'étude des organisations. Politique, (12),

111-120. https://doi.org/10.7202/040570ar

Ce document est protégé par la loi sur le droit d'auteur. L'utilisation des services d'Érudit (y compris la reproduction) est assujettie à sa politique d'utilisation que vous pouvez consulter en ligne.

https://apropos.erudit.org/fr/usagers/politique-dutilisation/ 


\section{ENTREVUE}

Michel Crozier et l'étude des organisations

Il y a dix ans, déjà, paraissait L'acteur et le système (Seuil), largement considéré depuis comme le principal ouvrage, en langue française, de théorie des organisations. Coauteur, avec Erhard Friedberg, de cette cuvre fondamentale, Michel Crozier reprend ici les bases de sa réflexion et la situe par rapport au paradigme de l'individualisme méthodologique, avant de commenter l'expérience néolibérale de cette décennie. Ses propos ont été recueillis à Paris en décembre 1986.

\section{De l'acteur au système}

"La méthode d'analyse des organisations que je préconise est très empirique tout en comportant, finalement, sa part de théorie. Inductive, elle aborde le social par le seul aspect que l'on puisse considérer comme une évidence, soit la façon dont les gens vivent leur situation. Bien avant la vogue de l'individualisme méthodologique, j'en suis venu à l'idée qu'on ne connaît les phénomènes sociaux, les phénomènes de groupe et les phénomènes d'organisation que par les individus. Il faut partir l'analyse de ce que les individus vous répètent, donc d'un premier point inductif. Mais il ne s'agit pas d'en rester à l'interactionnisme, à l'induction et au point de vue donné par l'individu. On ne s'intéressera pas non plus aux opinions ou aux attitudes en ce qu'elles seraient des prédictions de comportement. Plutôt, les témoignages des individus 
servent à reconstruire les rapports humains et le système d'organisation. Voici comment. On se concentre sur la déformation entre le point de vue donné par la personne et la réalité qu'elle vit. Cette déformation correspond à quelque chose de très essentiel dans le comportement humain, que nous appelons la stratégie. L'individu ne dit pas la vérité, il ne la connaît pas, il ne se l'avoue qu'à moitié à lui-même; l'individu nous révèle ses orientations.

Bien sûr, il ne s'agit pas de postuler une réalité objective; la réalité ne nous parvient jamais parfaitement puisqu'on ne peut la connaître qu'à travers ce que disent les personnes. Mais quand, dans une situation organisationnelle, vous avez plusieurs acteurs et même des groupes de personnes dans chaque position, on peut faire ressortir un certain nombre d'éléments objectifs par les recoupements d'entretiens.

Les témoignages ainsi recueillis servent à l'élaboration des hypothèses sur la stratégie. L'étape suivante consiste à réfléchir sur la rationalité de cette stratégie en retraçant les éléments du jeu, c'est-à-dire les éléments d'interdépendance qui relient les acteurs entre eux en raison de leurs stratégies réciproques. Ces jeux forment eux-mêmes des régulations de système à l'échelle de l'organisation, laquelle interagit avec son environnement immédiat, que nous appelons l'environnement pertinent. L'ensemble des régulations de système à l'intérieur de l'organisation et dans son rapport avec l'environnement pertinent forme le système d'action concret. C'est ainsi que par approximations successives, on va reconstruire un modèle d'organisation à partir à la fois de la confrontation des diverses stratégies de partenaires, de leurs relations de pouvoir quotidiennes et des contraintes qui sont les leurs.»

\section{Méthode ou théorie?}

«L'analyse stratégique, telle que je viens de la décrire, ne s'oppose pas nécessairement aux théories de l'organisation existantes 
mais elle remet en question l'essentiel de leurs résultats, car ces théories sont a priori abstraites et correspondent mal à la réalité. Il est juste de dire que l'analyse stratégique se prête mieux aux monographies, aux études de cas sur des organisations particulières, qu'aux réflexions extensives sur l'organisation générale. Je dis souvent que je ne peux apporter beaucoup de conclusions sur la théorie générale de l'organisation, mais que nos collègues qui théorisent largement en savent encore moins, parce qu'ils raisonnent de façon déductive et que leurs analyses empiriques ne visent qu'à démontrer la validité de leurs théories. Comment ne pas être frappé par la pauvreté de leurs résultats! Il n'y a qu'à penser aux enquêtes les plus célèbres et les plus imposantes comme celles du groupe d'Aston ou celle de Blau: tant d'années d'efforts et de moyens déployés pour démontrer, en somme, qu'on n'a rien démontré du tout puisque c'est l'hypothèse nulle qui s'est avérée la seule acceptable. Un autre exemple classique est celui des théories humanistes qui posaient que la permissivité rend les gens à la fois satisfaits, créatifs et efficaces. Il est difficile de résister à de telles hypothèses psychologiques car leur optimisme est très rassurant. Mais les résultats des analyses stratégiques que notre équipe avait pu mener nous ont bien obligés à admettre que les relations hiérarchiques sont beaucoup plus complexes que cela. Une autre déception célèbre a été l'engouement pour les déterminismes technologiques que tant de théoriciens voyaient, à tort, à la base des structures et des comportements organisationnels. La capacité prédictive de ces soi-disant déterminismes a fait long feu. Enfin, je mentionnerai l'échec des théories américaines de management qui ont été incapables de prendre en compte les contraintes fortes qui pèsent sur les organisations modernes.

Ma position est que pour les problèmes d'organisation comme pour beaucoup d'autres problèmes - l'état des recherches est trop peu avancé pour qu'on puisse valider des théories générales; 
la science, de toute façon, n'est pas la complétude ou la théorie fermée. J'ai toujours critiqué le scientisme extrême qui imprégnait la théorie des systèmes il y a quinze ans encore et qui, croyaiton, devait mener à une théorie définitive de l'organisation. L'analyse de système, telle que je la conçois, ne postule pas un systèmetype mais, au contraire, découvre des systèmes de relations de pouvoir à partir des réalités vécues dans les organisations et leurs environnements pertinents. L'analyse stratégique permet aussi d'étudier les phénomènes de changement et de porter un éclairage sur les modèles possibles d'intervention sur l'action humaine. C'est pourquoi je me suis tant intéressé aux problèmes d'apprentissage, de changement et de logiques de système. Il faut découvrir ces logiques à travers la grande obscurité des pouvoirs parallèles et autres arrangements cachés derrière les apparences formelles de l'organisation. »

\section{La rationalité ne connaît pas de frontières}

«L'individu, je le pose comme rationnel. Toute démarche scientifique est fondée sur le postulat de rationalité. Les formes de psychanalyse qui ont pour prétention de faire la science de l'irrationnel repoussent en fait la rationalité à un second ou à un troisième degré d'interprétation. La démarche d'analyse stratégique demande au chercheur de trouver la rationalité des comportements observés. Le chercheur trouve cette rationalité dans le contexte organisationnel qu'il va reconstruire comme un jeu. L'individu n'est jamais irrationnel, son comportement a toujours une logique, il est rationnel par rapport à des enjeux et des contraintes qu'il s'agit de découvrir.

Même les phénomènes culturels sont à interpréter à partir du postulat de rationalité. Les choix de valeurs ne se font pas au hasard. Les gens choisissent les valeurs qui leur conviennent en fonction de leur insertion dans une société, mais comme cette 
insertion est très complexe, les mêmes personnes adoptent des valeurs trop contradictoires pour que ces valeurs régissent leurs comportements. Actuellement, on me reproche d'imaginer un individu sans attache culturelle et privé de socialisation, alors que pendant très longtemps, on m'a accusé de l'inverse, soit d'avoir une vision culturaliste des organisations. Ces deux reproches opposés sont bien sûr injustifiés. La culture joue un rôle important, mais il ne faut pas en avoir une vue essentialiste. Sur ce plan, ma pensée a évolué depuis le phénomène bureaucratique (Seuil: 1964), mais même à cette époque, je ne partageais pas le point de vue culturaliste que les Américains, en particulier, m'ont prêté. D'ailleurs, ce livre ne portait pas sur la culture française, mais sur deux organisations particulières françaises dans lesquelles j'ai trouvé des caractéristiques culturelles fortes. Les hypothèses que j'ai pu formuler alors sur la centralisation et sur la crainte du face à face dans les relations interpersonnelles étaient - et me semblent encore - en bonne partie confirmées, mais en même temps, on en voit bien les limites. Les organisations françaises ont fortement évolué depuis vingt-cinq ans, même si les contraintes de système restent pour une part les mêmes.

Il faut se garder de tout culturalisme. Je prendrai à ce propos un exemple frappant que j'ai indirectement étudié: celui des paysans français. Les généralisations des années cinquante affirmaient très fortement l'image du paysan français individualiste, incapable de coopérer et réfractaire à toute modernisation. Or, toutes ces théories culturalistes se sont avérées fausses: les paysans français ont changé de façon absolument extraordinaire, sont devenus coopératifs et ont bâti des organisations beaucoup plus efficaces que les syndicats ouvriers, ce qui n'a pas empêché les traits culturels liés à la tradition catholique et à la petite propriété de subsister.

Je donnerai un autre exemple, plus contemporain, d'erreur culturaliste: le miracle japonais tel que couramment interprété en 
Occident. Actuellement, la fixation sur la notion de culture empêche de bien raisonner sur le Japon. Moi-même, j’ai presque commis cette erreur, tellement facile pour l'esprit, d'expliquer le Japon par sa différence culturelle au lieu de le voir comme un système rationnel en changement rapide depuis les années cinquante. Les généralisations sur le consensus japonais laissent croire que les Japonais vivent une rationalité sans drame et pensent comme un seul homme. En fait, leurs organisations aussi sont faites de relations de pouvoir; cependant, ils ont des méthodes de régulation extraordinairement plus efficaces que les nôtres. Il ne faut pas déduire de tout cela qu'il n'y a pas de contraintes culturelles; simplement, ces contraintes sont plus contingentes qu'on ne le croit.»

\section{À propos de l'individualisme méthodologique}

"De même que la prise en compte de la culture ne doit pas conduire à une vision essentialiste, la prise en compte de la rationalité ne doit pas dériver vers l'bomo economicus. Alors que l'individualisme méthodologique est un must comme présupposé de méthode, sa réduction à l'bomo economicus est d'une grande pauvreté et, pour cela, manque toute les réalités. Bien sûr, dans un premier temps, l'bomo economicus est une hypothèse séduisante qui permet de progresser à toute allure et de se battre contre les interprétations holistes basées sur la nation, la classe, le groupe, le mouvement social, ou sur d'autres de ces entités abstraites qui ont servi à masquer la réalité. Mais très vite, on se rend compte qu'il est tout à fait absurde de penser le comportement des hommes comme s'il pouvait se réduire au simple calcul économique.

L'individu est utilitariste si vous définissez l'utilitarisme comme la saisie des opportunités qui se présentent. En termes très généraux, le comportement de l'individu se lit comme suit: il cherche à tirer partie des opportunités sans prendre de risques exagérés, et il veut se placer aux bons endroits pour que ses mérites 
soient reconnus. Or, ces opportunités sont trop multiformes pour que l'on puisse les réduire à quelques hypothèses simplistes comme celles qu'a pu formuler l'école du public choice: l'élu censé ne vouloir que maximiser ses votes, le bureaucrate son budget, etc. Si l'école du public choice a été utile pour réfuter toutes les théories ésotériques sur l'essence de la bureaucratie, elle atteint elle-même ses limites très rapidement. Les enjeux sont tellement variés dans une bureaucratie, les interférences entre systèmes si complexes, que le public choice devient, forcément, à son tour, une théorie abstraite.

La règle principale de conduite des individus est l'utilisation de l'opportunité et non la maximisation du gain dans quelque domaine que ce soit. Dans une organisation, les opportunités naissent de toutes les zones d'incertitude, c'est-à-dire des failles qui subsistent toujours dans un système d'autorité, quel que soit son degré de formalisation. Les individus cherchent à mieux se placer dans le système de pouvoir afin d'obtenir des gains, certes, mais ces gains sont d'une infinie variété, à commencer par les satisfactions d'amour propre auxquelles les gens attachent une importance absolument formidable.»

\section{L'État comme organisation}

«Si les économistes se font habituellement une conception étroite de la rationalité, trop de politistes commettent la même erreur à propos du pouvoir. Ils raisonnent comme s'il y avait un système politique séparé du reste de la société. En réalité, toutes les organisations, qu'elles soient directement politiques ou non, sont faites de relations de pouvoir. Une idée que je combats avec beaucoup de respect pour l'adversaire - , est celle d'un État mythique qui serait d'une essence différente de toute autre réalité humaine. L'État est un ensemble d'organisations à étudier comme les autres phénomènes organisationnels. Il faut se défaire de ces grandes traditions européennes - la française, la prussienne - 
qui font de l'État quelque chose d'absolu. Il est remarquable que le postulat le plus répandu à propos de l'État, celui du monopole de la violence légitime, soit en fait une théorie politique de justification.

Il est non moins remarquable de constater que les explications par les idéologies globalisantes ont échoué à expliquer l'État comme elles avaient échoué à expliquer l'évolution des sociétés. Il faut bien constater que dans un système de marché poussé comme aux États-Unis, aussi bien que dans un système semi social-démocrate comme en Europe, on retrouve à peu près le même développement des formes étatiques. Dans les années soixante-dix, j'avais critiqué les théories planistes parce qu'elles sous-estimaient la capacité de l'individu de jouer de sa liberté pour échapper aux rationalisations auxquelles on voulait l'assujettir. De la même façon, je suis maintenant extrêmement critique vis-à-vis du néolibéralisme. Constater que les néolibéraux ont échoué partout équivaut maintenant à enfoncer une porte ouverte. L'expérience Reagan se solde par quelques succès et un beaucoup plus grand nombre d'échecs. L'expérience néolibérale française a avorté avant même d'avoir débuté, à l'exception des privatisations (qui viennent corriger une erreur des socialistes dont les dommages pour l'économie française étaient immédiatement appréciables). Je dirais que paradoxalement, bien qu'elle ait essuyé elle aussi sa part d'échecs, c'est Madame Thatcher qui a vraiment marqué de son empreinte une société en réunissant partiellement - on ne sait pas encore jusquà quel point - à casser le modèle de bureaucratie syndicaliste dans lequel s'enlisait l'Angleterre.

Pour l'essentiel, la marche en avant du néolibéralisme a tourné court parce que les problèmes ont été traités de façon doctrinaire et abstraite. Il faut lire l'ouvrage fascinant de David Stockman sur les déconvenues budgétaires des meilleurs des néolibéraux américains pour mesurer à quel point ces a priori théoriques 
n'avaient pas de prise sur la réalité. Quant aux Français, leur échec était inévitable car ils sont partis avec des doctrines fraîchement acquises plutôt que sur la base d'une connaissance sérieuse des réalités. J'ai simplement été surpris de la rapidité de l'effondrement.

Quand j'ai écrit, en 1979, On ne change pas la société par décret (Grasset), je visais surtout la gauche car c'est elle qui allait venir au pouvoir, mais qu'est-ce que la majorité actuelle a voulu faire avec l'Université, sinon d'essayer de la changer par décret? De la même façon pour la réforme de la SNCF, au lieu de réfléchir sérieusement à la complexité des problèmes d'organisation soulevés, le gouvernement s'est fié à son idéologie au point de vouloir imposer, du jour au lendemain, le libéralisme aux cheminots cégétistes. Le résultat désastreux obtenu était prévisible.»

\section{Quel avenir pour l'analyste stratégique?}

«Les analyses en termes de stratégies, de rationalités individuelles et de relations de pouvoir semblent se multiplier et on peut avoir l'impression qu'elles sont passées dans la sociologie courante. Je me réjouirais si tel était le cas. Malheureusement, l'analyse stratégique est trop souvent déformée en concepts abstraits, tandis que la pensée pratique, la seule qui compte, n'en est nullement affectée.

J'essaye de réfléchir actuellement aux raisons profondes de cette difficulté de compréhension. J'en arrive à croire que les concepts pourtant simples que j'ai élaborés touchent à des habitudes de raisonnement très difficiles à changer parce que profondément intériorisées. Passer de la conception du pouvoir comme attribut à la conception du pouvoir comme relation, ce qui était le point de départ pour moi tout simple de l'analyse stratégique, a été tout juste un point d'arrivée pour Michel Foucault qui en a découvert l'intérêt dans les dernières années de sa vie. Sera-t-il 
un nouveau point de départ pour Pierre Bourdieu qui le découvre à son tour maintenant? Je l'espère, mais que de temps perdu!

Un immense effort me paraît indispensable désormais pour remettre en question les modèles de rationalité beaucoup trop étroits hérités de Hegel et que quelqu'un comme Alexandre Kojeve a répandus en France dans les années cinquante.

L'individualisme méthodologique ne constitue absolument pas l'antidote nécessaire. Tel qu'il est développé actuellement, il me paraît extrêmement étroit, lui aussi. Il pousse inconsciemment à un réductionnisme utilitariste, efficace certes, mais qui trouve très rapidement ses limites sans que la méthode puisse donner les moyens de les apprécier.

Je m'aperçois avec le recul du temps que je n'ai été ni assez clair pour être vraiment compris ni assez ambitieux pour apporter la contribution théorique indispensable qui aurait dû appuyer ma pratique. Ce n'était évidemment pas possible car non seulement je n'étais pas équipé pour résoudre le problème, mais j'étais incapable même de concevoir son existence. J'espère avoir le temps de me consacrer, dans les années qui viennent, à cette reprise philosophique.

En attendant, j'ai essayé dans mon nouveau livre État modeste, État moderne (Fayard: 1987), de faire réfléchir les élites françaises administratives, politiques et plus généralement technocratiques sur le caractère tout à fait archaïque des modèles de rationalité qui sont sous-jacents à leurs modes de décision et de gouvernement. C'est cet archaïsme qui permet d'expliquer l'extraordinaire répétition d'erreurs commises aussi bien à droite qu'à gauche et que nous devons constater. Le problème bien sûr ne se limite pas du tout à la France. Tout l'Occident vit une crise profonde de ce point de vue. Mais c'est en France que je travaille et c'est pour le cas français que j'essaie d'élaborer une stratégie pour un autre changement.» 